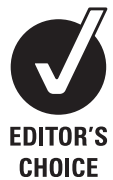

CHOICE
- An additional table, figure and appendix are published online only. To view these files please visit the journal online (http:// thorax.bmj.com).

1 Department of Medicine, Johns Hopkins University School of Medicine, Baltimore,

Maryland, USA

${ }^{2}$ Clinical Pharmacology, School of Medicine, Health Policy and Practice, University of East Anglia, Norwich, UK ${ }^{3}$ Division of Public Health Sciences, Wake Forest University School of Medicine, Winston-Salem, North Carolina, USA

\section{Correspondence to} Sonal Singh, Department of Medicine, Johns Hopkins University School of Medicine, 1830 E Monument St, Suite 8063, Baltimore, MD 21287, USA; ssingh31@jhu.edu

SS and YKL contributed equally.

Received 4 October 2010 Accepted 6 January 2011 Published Online First 15 February 2011

\title{
Long-term use of thiazolidinediones and the associated risk of pneumonia or lower respiratory tract infection: systematic review and meta-analysis
}

\author{
Sonal Singh, ${ }^{1}$ Yoon K Loke, ${ }^{2}$ Curt D Furberg ${ }^{3}$
}

\section{ABSTRACT}

Introduction The peroxisome proliferator-activated receptor- $\gamma$ agonists rosiglitazone and pioglitazone activate glucocorticoid receptors and have an immunomodulatory effect. The authors aimed to systematically determine the risk of pneumonia or lower respiratory tract infections associated with thiazolidinediones.

Methods Systematic searches of MEDLINE, EMBASE, regulatory documents and trial registries were carried out for randomised controlled trials of thiazolidinediones with no date restrictions through March 2010. The authors selected long-term ( $\geq 1$ year) randomised controlled trials of thiazolidinediones versus a placebo, metformin or sulfonylurea control for prevention or treatment of type 2 diabetes that reported on pneumonia or lower respiratory tract infection adverse events or serious adverse events (hospitalisation, disability or death). Relative risks (RRs) were estimated using a fixed-effects meta-analysis, and statistical heterogeneity was assessed using the $\mathrm{I}^{2}$ statistic.

Results Thirteen trials $(n=17627$, including 8163 patients receiving thiazolidinediones and 9464 patients receiving control therapy) with a duration of follow-up of $1-5.5$ years were included after a detailed screening of 58 studies. Thiazolidinediones were associated with a statistically significantly increased risk for any pneumonia or lower respiratory tract infection ( $n=130 / 8163$ vs $100 / 9464 ;$ RR $1.40 ; 95 \%$ Cl 1.08 to $\left.1.82 ; p=0.01 ; l^{2}=0 \%\right)$ and serious pneumonia or lower respiratory tract infection $(n=111 / 7391$ vs $87 / 8692$; RR 1.39; $95 \%$ Cl 1.05 to $1.83 ; p=0.02 ;\left.\right|^{2}=0 \%$ ). Interpretation Long-term thiazolidinedione use is associated with a modestly increased risk of any pneumonia or lower respiratory tract infection and serious pneumonia or lower respiratory tract infection in patients with type 2 diabetes.

\section{INTRODUCTION}

Thiazolidinediones (rosiglitazone and pioglitazone) are peroxisome proliferator-activated receptor (PPAR) $\gamma$ agonists. Rosiglitazone is a PPAR $\gamma$ agonist whereas pioglitazone has both $\alpha$ and $\gamma$ effects. PPAR activation has anti-inflammatory and immunomodulatory effects in the lung. ${ }^{1}$ Thiazolidinediones act as partial agonists at the glucocorticoid receptor. ${ }^{2}$ Rosiglitazone significantly improved lung function compared with inhaled beclometasone in a recent randomised controlled trial (RCT) in people who smoke and have asthma. ${ }^{3}$ Pioglitazone is as effective as dexamethasone in reducing airway hyper-responsiveness and pulmonary inflammatory mediators in animal studies. ${ }^{4}$ There is considerable optimism about the potential of the thiazolidinediones to favourably affect the course of asthma and chronic obstructive pulmonary disease.

The long-term effects of thiazolidinediones, however, are unpredictable, resulting in unusual toxicities. There have been concerns about an increased risk of myocardial infarction associated with rosiglitazone. ${ }^{5-7}$ Thiazolidinediones are associated with an increased risk of congestive heart failure, $^{6-8}$ fractures (in women), ${ }^{9}$ and possibly macular oedema.

It is possible that these immunomodulatory and glucocorticoid-like properties of the thiazolidinediones could potentially induce susceptibility to lung infections, similar to glucocorticoids in chronic obstructive pulmonary disease. ${ }^{10}$ A signal for an increased risk of pneumonia in patients with type 2 diabetes was reported in a long-term trial of pioglitazone. ${ }^{11}$ Another long-term trial of rosiglitazone reported a non-significant increase in pneumonia adverse events. ${ }^{6}$ However, the exact risk of rare, serious adverse events such as pneumonia cannot always be conclusively determined from a single trial because of inadequate statistical power. The authors aimed to systematically determine the risk of pneumonia or lower respiratory tract infection adverse events associated with thiazolidinedione therapy in long-term clinical trials of patients with type 2 diabetes.

\section{METHODS}

Selection of trials with pneumonia or lower respiratory tract infection adverse event data

Our specific inclusion criteria were as follows: a study design consisting of parallel group RCTs of any thiazolidinedione (rosiglitazone or pioglitazone) with a treatment duration of at least 12 months; study participants with type 2 diabetes mellitus; control treatment which could be placebo, metformin or sulfonylurea, with the only difference between the treatment groups being thiazolidinedione exposure among participants; studies that reported data on 'pneumonia' or 'lower respiratory tract infection' adverse events or serious adverse events (including 0 events).

Active oral hypoglycaemic agents (metformin or sulfonylurea) were used as active comparators because metformin and sulfonylurea are not known to increase the risk of pneumonia. The recently approved incretin mimetics have been linked to an increase in the risk of infections. ${ }^{12}$ The analysis was restricted to RCTs of at least 12 months' duration to evaluate the risk of pneumonia associated with long-term use of thiazolidinediones. 


\section{Search strategy}

The authors evaluated all the RCTs identified from the database searches (MEDLINE, EMBASE, Cochrane Controlled Trials Register) in their previous systematic reviews. ${ }^{7-9}$ The authors (YKL and SS) conducted an updated search of MEDLINE and EMBASE (Ovid SP from June 2008 to March 2010) for RCTs of rosiglitazone and pioglitazone. Trial reports were evaluated of all phase 3 and 4 published or unpublished trials of the GlaxoSmithKline clinical trials register, the Takeda clinical trials register and the Clinical Study Results Database for additional RCTs. Existing systematic reviews and the bibliographies of the studies that were included were checked. The search was limited to English-language articles, and included pre-approval and unpublished studies from regulatory documents and trial registries. The updated search strategy is outlined in online appendix S1.

\section{Study selection}

Trials were identified and subjected to inclusion and exclusion criteria. Two reviewers (SS and YKL) independently and in duplicate scanned all titles and abstracts that indicated the study was an RCT evaluating the use of thiazolidinediones among patients with type 2 diabetes. After obtaining full reports of potentially relevant trials, the same reviewers independently assessed eligibility from full-text articles.

\section{Study characteristics}

A prespecified protocol was used to record the location and duration of the RCT (in years), the inclusion and exclusion criteria, the dose and frequency of thiazolidinedione and control interventions, mean age and sex of participants, and mean glycated haemoglobin levels at baseline.

\section{Risk of bias assessment}

The Cochrane Toolkit was used for the assessment of bias in evaluating each trial for the reporting of sequence generation, allocation concealment, the use of blinding of participants and personnel, and information on loss to follow-up or withdrawal rates. ${ }^{13}$ In accordance with the Cochrane handbook of systematic reviews, the authors assessed the strength of adverse events data by noting how the investigators monitored and recorded adverse events. ${ }^{13}$

\section{Outcome measures}

The primary prespecified outcomes measures were any pneumonia or lower respiratory tract infection adverse event (irrespective of severity), and pneumonia or lower respiratory tract infection reported as serious adverse events. Serious adverse events are life-threatening, require hospitalisation, or lead to significant disability or death.

\section{Data abstraction}

Two reviewers (SS and YKL), independently and as a team, assessed the eligibility and quality of studies for reporting of adverse events. They separately extracted data (including 0 events) on the proportion of patients with any pneumonia or lower respiratory tract infection among tabulated listings of adverse events for each study. Published reports were reconciled with trials in the clinical trials register when possible to avoid potential duplication. If there were multiple reports available for a particular study, data on pneumonia were extracted from the latest version.

Two reviewers (SS and YKL) were independently involved in all stages of study selection, data extraction and quality assessment. Any discrepancies were resolved through discussion. The reviewers achieved consensus on inclusion of studies and data extraction.

\section{Data synthesis}

Review Manager (RevMan), version 5.024 (Nordic Cochrane Center, Copenhagen, Denmark) was used to conduct a metaanalysis of the pooled relative risks (RRs), with 95\% CI, using the fixed-effects model for dichotomous outcomes. The fixedeffects Mantel-Haenszel model is the appropriate method for meta-analysis of rare events because the random effects model lacks statistical power. ${ }^{14}$ A continuity correction of 0.5 was used when there were zero events in one intervention arm. All reported $p$ values are two sided, with significance set at $p<0.05$. Statistical heterogeneity was assessed using the $\mathrm{I}^{2}$ statistic. ${ }^{15} \mathrm{~A}$ substantial level of heterogeneity is indicated by $\mathrm{I}^{2}$ values of $50 \%$ or more. ${ }^{15}$

Prespecified sensitivity analyses were conducted to determine the influence of statistical models (use of Peto odds ratio which has been recommended for analysis of rare events, or the random effect which has less power ${ }^{14}$ on effect size. A subgroup analysis was also conducted to evaluate for intraclass differences in the risk of pneumonia between rosiglitazone and pioglitazone. Publication bias was assessed by visual inspection for funnel plot asymmetry, ${ }^{13}$ and evaluation of effect sizes in published studies versus data obtained from unpublished studies. The fail-safe number using the Rosenberg method was estimated to assess the potential impact of missing data in the meta-analysis (ie, the number of additional negative trials that would be required to reverse a significant finding in the meta-analysis). ${ }^{16}$

The number needed to harm (and 95\% CI) was estimated by applying the pooled RR from the meta-analysis to the average control event rate in different populations. The number needed to harm is the number of patients with type 2 diabetes who need to be treated with a thiazolidinedione, rather than with placebo or comparators, for one additional patient to have pneumonia or a lower respiratory tract infection.

\section{RESULTS}

The selection of trials is shown in figure 1 (online). The authors identified 280 potentially relevant citations, and after a detailed review of 58 studies, selected 10 datasets involving 13 trials of 17627 patients that reported on pneumonia or lower respiratory tract infections. ${ }^{6} 11$ 17-32

Trial characteristics are shown in table 1. In the trials 8163 participants received thiazolidinediones and 9464 received control therapy. Nine RCTs evaluated thiazolidinediones against active controls, ${ }^{6}$ 17-21 27-32 while four RCTs evaluated them against placebo. ${ }^{11} 22-26$ The RCTs ranged in duration from 1 year to 5.5 years $^{6}$ and the sample size ranged from as low as $57^{26}$ to $5238 .^{11}$ Thiazolidinedione-exposed participants had similar glycated haemoglobin at baseline to the control patients. Eight RCTs evaluated rosiglitazone. ${ }^{6}{ }^{17-27}$ Five RCTs evaluated pioglitazone, including one large RCT (the Prospective Pioglitazone Clinical Trial in Macrovascular Events, PROactive) ${ }^{11}$ and another dataset, ${ }^{28}$ which reported a pooled analysis among older patients with diabetes from four pioglitazone trials. ${ }^{29-32}$

Trial quality was generally satisfactory (online table S1). All the RCTs were double blind except for the Rosiglitazone Evaluated for Cardiovascular Outcomes and Regulation of Glycaemia in Diabetes (RECORD) trial, which was open label. ${ }^{6}$ Four trials had inadequate reporting of allocation concealment, sequence generation or loss to follow-up. ${ }^{21} 30-32$ Eight RCTs were judged to be at low risk of bias (adequate 


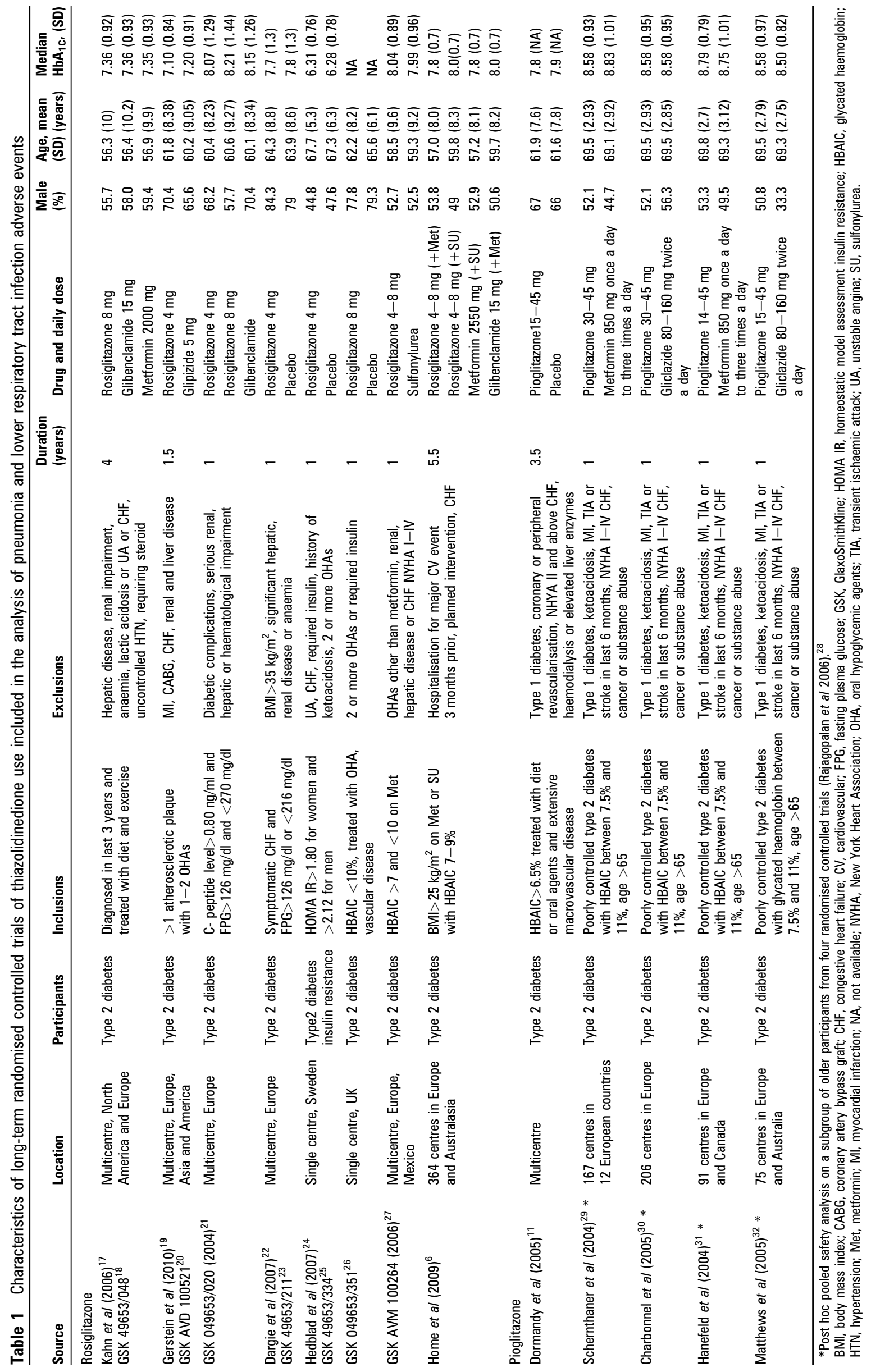


sequence generation, allocation concealment and double blinding, and clear reporting of loss to follow-up or withdrawal rates), whereas the remaining five RCTs were at unclear risk of bias. $62130-32$

Because pneumonia and lower respiratory tract infection have not previously been linked to thiazolidinedione therapy, detection in these trials occurred via routine monitoring of adverse events or serious adverse events because it is not possible to prespecify diagnostic criteria for unexpected adverse events. Accordingly, none of the included RCTs had objective pneumonia definitions, or reported the proportion of patients that had radiographic confirmation of pneumonia. Thus the methods for diagnosing pneumonia and lower respiratory tract infection possibly varied between trials (clinical vs radiographical). Seven trials reported pneumonia or lower respiratory tract infection to be a serious adverse event, ${ }^{611} 17-23$ whereas six trials reported lower respiratory tract infection as an adverse event. ${ }^{26-32}$ Approximately $86 \%$ of pneumonia and lower respiratory tract infection events in our meta-analysis were classified as serious adverse events leading to hospitalisation, disability or death. Although six trials did not report on cause-specific mortality, ${ }^{6} 1128-32$ available information on fatal adverse events from seven rosiglitazone trials ${ }^{17}$ reported no deaths due to pneumonia or lower respiratory tract infection associated with rosiglitazone. Thus it is possible that most of these serious adverse events caused by pneumonia or lower respiratory tract infection stemmed from hospitalisation.

\section{Risk of pneumonia or lower respiratory tract infection}

Thiazolidinediones were associated with a significantly increased risk of pneumonia or lower respiratory tract infection compared with controls in a meta-analysis of 13 RCTs involving 17627 participants ( $\mathrm{n}=130 / 8163$ vs $100 / 9464$; $1.59 \%$ vs $1.06 \%$; RR 1.40 ; $95 \%$ CI 1.08 to $1.82 ; \mathrm{p}=0.01$ ); there was no statistical heterogeneity among the included trials $\left(\mathrm{I}^{2}=0 \%\right)$ (figure 2). ${ }^{6} 1117-32$

\section{Risk of pneumonia or lower respiratory tract infection as serious adverse events}

Thiazolidinediones were associated with a significantly increased risk of serious adverse events caused by pneumonia or lower respiratory tract infections compared with controls in a meta-analysis of seven trials involving 16,083 patients (RR 1.39; $95 \%$ CI 1.05 to 1.83; $\mathrm{p}=0.02$ ); there was no statistical heterogeneity among the included trials $\left(\mathrm{I}^{2}=0 \%\right)$ (figure 3$) .{ }^{6} 11 \quad 17-23$

\section{Sensitivity analysis}

The sensitivity analysis using the Peto odds ratio yielded estimates similar to those of the fixed-effects model for the risk of any pneumonia or lower respiratory tract infection associated with the thiazolidinediones (RR 1.42; 95\% CI 1.09 to 1.84; $\left.\mathrm{p}=0.009 ; \mathrm{I}^{2}=0 \%\right) .{ }^{6} 1117-32$
The sensitivity analysis using a random-effects model yielded estimates similar to those of the fixed-effects model for the risk of any pneumonia or lower respiratory tract infection associated with the thiazolidinediones (RR 1.38; 95\% CI 1.06 to 1.79 ; $\left.\mathrm{p}=0.02 ; \mathrm{I}^{2}=0 \%\right)^{6}{ }^{6} 11 \quad 17-32$

The direction of effect for both the thiazolidinediones showed a similar increased risk, with the RRs for pioglitazone achieving statistical significance (RR 1.63; 95\% CI 1.09 to 2.46; $\mathrm{p}=0.02$ ) in a meta-analysis of five trials involving 6129 patients. ${ }^{11}{ }^{28-32}$ The RRs for rosiglitazone did not reach statistical significance (RR 1.26 ; $95 \%$ CI 0.90 to 1.76 ; $p=0.23$ ) in a meta-analysis of eight trials involving 11498 patients, but a clinically significant excess risk could not be ruled out. ${ }^{6} 17-27$ The test for homogeneity of effects across the two thiazolidinediones pioglitazone and rosiglitazone did not demonstrate a statistically significant difference between the two agents $(p=0.32)$. However, these subgroup analyses should be interpreted for the direction of effect, rather than the magnitude of statistical significance, because subgrouping or removal of specific studies can render the meta-analysis underpowered to detect differences.

\section{Assessment of publication bias and missing data}

Visual inspection of the funnel plot did not reveal any evidence of asymmetry (online figure S1). The RR for pneumonia from unpublished data was 1.38 (95\% CI 0.82 to 2.30), which was similar to that of the published studies (RR 1.40; 95\% CI 1.05 to 1.90). To reverse the significantly increased risk of pneumonia or lower respiratory tract infection shown in our meta-analysis, 11 negative studies with an average sample size of 1400 participants would be required. ${ }^{23}$

\section{Estimated number needed to harm}

Using estimates of the incidence of pneumonia of 4919/100 000 person-years from a population-based study (mean age 61 years, $51.7 \%$ male) in patients with type 2 diabetes in Ontario, ${ }^{33}$ the number needed to harm for pneumonia or lower respiratory tract infection associated with thiazolidinedione use was estimated to be 55 (95\% CI 28 to 269). Treatment of 55 patients with thiazolidinediones for 3.7 years would result in one extra case of pneumonia or lower respiratory tract infection based on the event rate in population-based studies. Based on the average control event rate in the trials, the estimated number needed to harm for any pneumonia or lower respiratory tract infection associated with thiazolidinedione use is 239 (95\% CI 117 to 1191).

\section{DISCUSSION}

Our meta-analysis of 17627 patients raises serious concern about a significant association between long-term thiazolidinedione use and pneumonia or lower respiratory tract infection leading to adverse events or serious adverse events among
Figure 2 Fixed-effects meta-analysis of long-term randomised controlled trials of pneumonia or lower respiratory tract infection with thiazolidinediones versus controls.

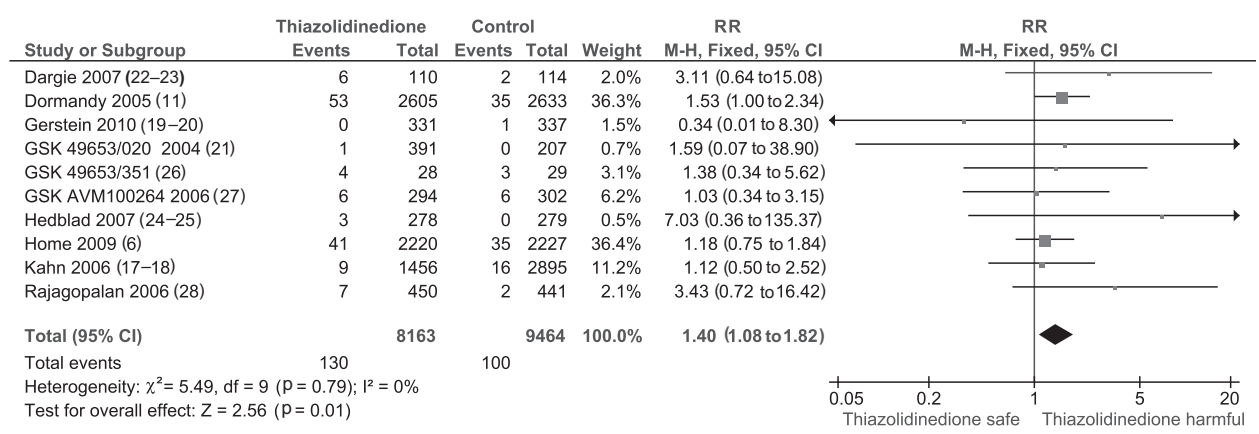


Figure 3 Fixed-effects meta-analysis of long-term randomised controlled trials of serious pneumonia or lower respiratory tract infection with thiazolidinediones versus controls.

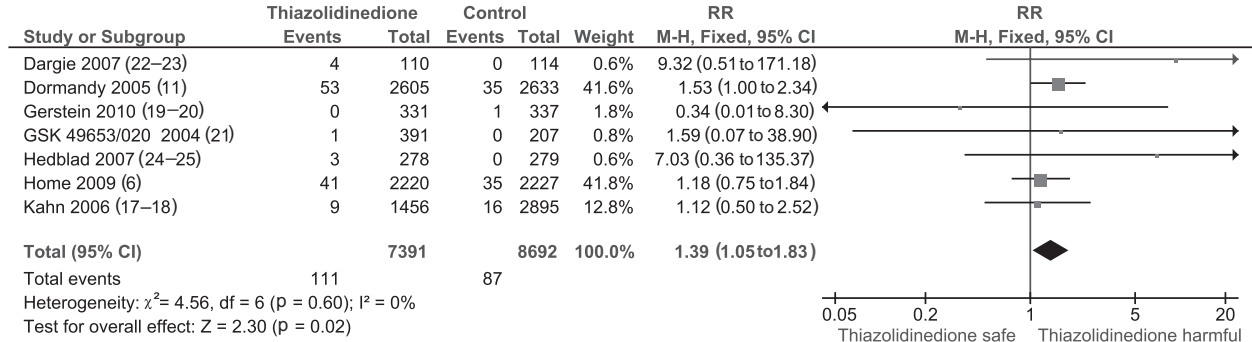

analysis ought to be conducted to determine any differences in the risk among people who smoke, people with chronic obstructive pulmonary disease, or those vaccinated with the influenza or pneumococcal vaccine. Clinical trials of thiazolidinediones among patients with type 2 diabetes, and ongoing trials in patients with obstructive lung disease, should monitor the risk of pneumonia using objective definitions with radiological and microbiological confirmation. The safety concern for long-term thiazolidinedione use needs to be replicated in carefully conducted epidemiological studies.

\section{CONCLUSION}

Long-term thiazolidinedione use is associated with a modestly increased risk of pneumonia or lower respiratory tract infection, and serious pneumonia or lower respiratory tract infection in patients with type 2 diabetes.

Funding SS is supported by the Johns Hopkins Clinical Research Scholars Program. This publication was made possible by Grant Number 1KL2RR025006-03 from the National Center for Research Resources (NCRR), a component of the National Institutes of Health (NIH), and NIH Roadmap for Medical Research. Its contents are solely the responsibility of the authors and do not necessarily represent the official view of NCRR or NIH. Information on NCRR is available on the website (http://www. ncrr.nih.gov/). Information on Re-engineering the Clinical Research Enterprise can be obtained from the website (http://nihroadmap.nih.gov/clinicalresearch/overviewtranslational.asp). The design and conduct of the study; collection, management, analysis, and interpretation of the data; and preparation, review, or approval of the manuscript was independent of any other sources of funding. Other Funders: $\mathrm{NIH}$.

Competing interests Dr Furberg reports that he is a senior investigator and steering committee chair of the National Health, Lung, and Blood Institute-sponsored Cardiovascular Health Study (CHS), a cohort study to examine risk factors of coronary heart disease and stroke in the elderly. In 2004, the study received a grant for $\$ 280000$ from GlaxoSmithKline to determine the relationships between LPPLA-2 and various vascular diseases. The grant was primarily for the CHS central laboratory and the CHS coordinating center. Funding was channeled through Wake Forest University and Dr Furberg was the CHS principal investigator, but he received no salary support and the funding period ended 30 June 2006. Dr Furberg did not benefit personally from the grant and has no funding from any other manufacturers of antidiabetic medications. No other financial disclosures were reported. No specific financial interests and relationships and affiliations relevant to the subject of their manuscript were reported by Sonal Singh and Yoon Loke.

Contributors Dr Singh had full access to all of the data in the study and takes responsibility for the integrity of the data and the accuracy of the data analysis Study concept and design: Singh and Loke. Acquisition of data: Singh and Loke. Analysis and interpretation of data: Singh, Loke, Furberg. Drafting of the manuscript: Singh, Loke, Furberg. Critical revision of the manuscript for important intellectual content: Singh, Loke, Furberg Statistical analysis: Singh and Loke. Administrative, technical, and material support: Singh and Loke. Study supervision: Furberg and Loke.

Provenance and peer review Not commissioned; externally peer reviewed.

\section{REFERENCES}

1. Belvisi MG, Mitchell JA. Targeting PPAR receptors in the airway for the treatment of inflammatory lung disease. Br J Pharmacol 2009;158:994-1003.

2. Matthews L, Berry A, Tersigni M, et al. Thiazolidinediones are partial agonists for the glucocorticoid receptor. Endocrinology 2009;150:75-86.

3. Spears M, Donnelly I, Jolly L, et al. Bronchodilatory effect of the PPAR-gamma agonist rosiglitazone in smokers with asthma. Clin Pharmacol Ther 2009;86:49-53. tigation. Individual patient data analysis could yield further information on the risk of pneumonia or lower respiratory tract infection with long-term use of thiazolidinediones. Stratified 
4. Narala VR, Ranga R, Smith MR, et al. Pioglitazone is as effective as dexamethasone in a cockroach allergen-induced murine model of asthma. Respir Res 2007;8:90.

5. Nissen SE, Wolski K. Effect of rosiglitazone on the risk of myocardial infarction and death from cardiovascular causes. N Engl J Med 2007:356:2457-71.

6. Home PD, Pocock SJ, Beck-Nielsen H, et al; RECORD Study Team. Rosiglitazone evaluated for cardiovascular outcomes in oral agent combination therapy for type 2 diabetes (RECORD): a multicentre, randomised, open-label trial. Lancet 2009;373:2125-35.

7. Singh S, Loke YK, Furberg CD. Long-term risk of cardiovascular events with rosiglitazone: a meta-analysis. JAMA 2007;298:1189-95.

8. Singh S, Loke YK, Furberg CD. Thiazolidinediones and heart failure: a teleo-analysis. Diabetes Care 2007:30:2148-53.

9. Loke YK, Singh S, Furberg CD. Long-term use of thiazolidinediones and fractures in type 2 diabetes: a meta-analysis. CMAJ 2009;180:32-9.

10. Singh S, Amin AV, Loke YK. Long-term use of inhaled corticosteroids and the risk of pneumonia in chronic obstructive pulmonary disease-a meta-analysis. Arch Intern Med 2009:169:219-29.

11. Dormandy JA, Charbonnel B, Eckland DJ, et al; PROactive Investigators. Secondary prevention of macrovascular events in patients with type 2 diabetes in the PROactive Study (PROspective pioglitAzone Clinical Trial in macroVascular Events): a randomized controlled trial. Lancet 2005;366:1279-89.

12. Amori RE, Lau J, Pittas AG. Efficacy and safety of incretin therapy in type 2 diabetes: systematic review and meta-analysis. JAMA 2007:298:194-206.

13. Loke YK, Price D, Herxheimer A. Adverse effects. In: Higgins JPT, Green S, eds. Cochrane Handbook for Systematic Reviews of Interventions Version 5.0.0 [Updated February 2008]. Chichester (UK): The Cochrane Collaboration, 2008. http://www. cochrane-handbook.org (accessed 15 Dec 2010)

14. Bradburn MJ, Deeks JJ, Berlin JA, et al. Much ado about nothing: a comparison of the performance of meta-analytical methods with rare events. Stat Med 2007; 26:53-77.

15. Higgins JP, Thompson SG, Deeks JJ, et al. Measuring inconsistency in meta-analyses. BMJ 2003;327:557-60.

16. Rosenberg MS. The file-drawer problem revisited: a general weighted method for calculating fail-safe numbers in meta-analysis. Evolution 2005:59:464-8.

17. Kahn SE, Haffner SM, Heise MA, et al; ADOPT Study Group. Glycemic durability of rosiglitazone, metformin, or glyburide monotherapy. $N$ Engl J Med 2006;355:2427-43.

18. GlaxoSmithKline Clinical Trials Register. A randomized, double-blind study to compare the durability of glucose lowering and preservation of pancreatic beta-cell function of rosiglitazone monotherapy compared to metformin or glyburide/ glibenclamide in patients with drug-naive, recently diagnosed type 2 diabetes mellitus. Brentford (UK): GlaxoSmithKline 49653/048, 2007. http://www.gskclinicalstudyregister.com/files/pdf/20387.pdf (accessed 15 Dec 2010)

19. Gerstein HC, Ratner RE, Cannon CP, et al; APPROACH Study Group. Effect of rosiglitazone on progression of coronary atherosclerosis in patients with type 2 diabetes mellitus and coronary artery disease: the assessment on the prevention of progression by rosiglitazone on atherosclerosis in diabetes patients with cardiovascular history trial. Circulation 2010;121:1176-87.

20. GlaxoSmithKline Clinical Trials Register. A phase III, 18 month, multicenter randomized, double-blind, active-controlled clinical trial to compare rosiglitazone versus glipizide on the progression of atherosclerosis in subjects with type 2 diabetes mellitus and cardiovascular disease (APPROACH)/AVD 100521, 2010. Brentford (UK): GlaxoSmithKline. http://www.gsk-clinicalstudyregister.com/files/pdf/20512.pdf (accessed 15 Dec 2010).

21. GlaxoSmithKline Clinical Trials Register. A multicentre, double-blind, parallel group comparative study to evaluate the efficacy, safety and tolerability of rosiglitazone versus glibenclamide therapy, when administered to patients with type 2 diabetes mellitus. Brentford (UK): GlaxoSmithKline 49653/020, 2004 http://www. gsk-clinicalstudyregister.com/files/pdf/2834.pdf (accessed 15 Dec 2010)

22. Dargie HJ, Hildebrandt PR, Riegger GA, et al. A randomized, placebo-controlled tria assessing the effects of rosiglitazone on echocardiographic function and cardiac status in type 2 diabetic patients with New York Heart Association functional class or II heart failure. J Am Coll Cardiol 2007:49:1696-704

23. GlaxoSmithKline Clinical Trials Register. A 52-week double-blind study of the effect of rosiglitazone on cardiovascular structure and function in subjects with type 2 diabetes mellitus and congestive heart failure (NYHA class I/II). Brentford (UK): GlaxoSmithKline 49653/211, 2005. http://www.gsk-clinicalstudyregister.com/files/ pdf/23019.pdf (accessed 15 Dec 2010).

24. Hedblad B, Zambanini A, Nilsson P, et al. Rosiglitazone and carotid IMT progression rate in a mixed cohort of patients with type 2 diabetes and the insulin resistance syndrome: main results from the Rosiglitazone Atherosclerosis Study. J Intern Med 2007:261:293-305.

25. GlaxoSmithKline Clinical Trials Register. Brentford (UK): GlaxoSmithKline. 49653/334. http://www.gsk-clinicalstudyregister.com/files/pdf/20391.pdf (accessed 15 Dec 2010).

26. GlaxoSmithKline Clinical Trials Register. Rosiglitazone and plaque study: a 12 month randomised, double-blind, placebo controlled, magnetic resonance imaging study to evaluate the effect of rosiglitazone on the structure and composition of carotid atherosclerotic plaques in subjects with type 2 diabetes mellitus and coexisting vascular disease or hypertension. Brentford (UK): GlaxoSmithKline, 49653/351, 2008. http://www.gsk-clinicalstudyregister.com/files/pdf/23025.pdf (accessed 15 Dec 2010)

27. GlaxoSmithKline Clinical Trials Register. A randomised, multi-centre, phase $\mathrm{N}$, double-blind, parallel group study comparing the effects of 52 weeks' administration of AVANDAMET and metformin plus sulphonylurea on change in $\mathrm{HbA1c}$ from baseline in overweight type 2 diabetics poorly controlled on metformin [study no AVM100264]. Brentford (UK): GlaxoSmithKline AVM 100264, 2008. http://gsk-clinicalstudyregister com/files/pdf/20518.pdf (accessed 15 Dec 2010).

28. Rajagopalan R, Xu Y, Abbadessa M, et al; Quartet Study Group. The effect of pioglitazone on glycemic and lipid parameters and adverse events in elderly patients with type 2 diabetes mellitus: a post hoc analysis of four randomized trials. Am J Geriatr Pharmacother 2006:4:123-33.

29. Schernthaner G, Matthews DR, Charbonnel B, et al; QUARTET Study Group. Efficacy and safety of pioglitazone versus metformin in patients with type 2 diabetes mellitus: a double-blind, randomized trial. J Clin Endocrinol Metab 2004:89:6068-76.

30. Charbonnel BH, Matthews DR, Schernthaner G, et al; QUARTET Study Group. A long-term comparison of pioglitazone and gliclazide in patients with type 2 diabetes mellitus: a randomized, double-blind, parallel-group comparison trial. Diabet Med 2005;22:399-405.

31. Hanefeld M, Brunetti $P$, Schernthaner GH, et al; QUARTET Study Group. One-year glycemic control with a sulfonylurea plus pioglitazone versus a sulfonylurea plus metformin in patients with type 2 diabetes. Diabetes Care 2004:27:141-7.

32. Matthews DR, Charbonnel BH, Flanefeld M, et al. Long-term therapy with addition of pioglitazone to metformin compared with the addition of gliclazide to metformin in patients with type 2 diabetes: a randomized, comparative study. Diabetes Metab Res Rev 2005;21:167-74.

33. Shah BR, Hux JE. Quantifying the risk of infectious diseases for people with diabetes. Diabetes Care 2003;26:510-13.

34. Hall JM, McDonnell DP. The molecular mechanisms underlying the proinflammatory actions of thiazolidinediones in human macrophages. Mol Endocrinol 2007;21:1756-68.

35. Bolen S, Feldman L, Vassy J, et al. Systematic review: comparative effectiveness and safety of oral medications for type 2 diabetes mellitus. Ann Intern Med 2007;147:386-99.

Thorax online

Visit Thorax online and listen to the latest podcast, post comments and download any you might have missed. Keep informed and up to date by visiting thorax.bmj.com. 